Article

\title{
Compact Trap-Assisted-Tunneling Model for Line Tunneling Field-Effect-Transistor Devices
}

\author{
Faraz Najam $1 D$ and Yun Seop Yu *(D) \\ ICT \& Robotics Engineering and IITC, Hankyong National University, Anseong 17579, Korea; \\ faraznajam@hknu.ac.kr \\ * Correspondence: ysyu@hknu.ac.kr; Tel.: +81-31-670-5293
}

Received: 25 May 2020; Accepted: 24 June 2020; Published: 28 June 2020

check for updates

\begin{abstract}
Trap-assisted-tunneling (TAT) is a well-documented source of severe subthreshold degradation in tunneling field-effect-transistors (TFET). However, the literature lacks in numerical or compact TAT models applied to TFET devices. This work presents a compact formulation of the Schenk TAT model that is used to fit experimental drain-source current $\left(I_{\mathrm{ds}}\right)$ versus gate-source voltage $\left(V_{\mathrm{gs}}\right)$ data of an L-shaped and line tunneling type TFET. The Schenk model incorporates material-dependent fundamental physical constants that play an important role in influencing the TAT generation $\left(G_{\mathrm{TAT}}\right)$ including the lattice relaxation energy, Huang-Rhys factor, and the electro-optical frequency. This makes fitting any experimental data using the Schenk model physically relevant. The compact formulation of the Schenk TAT model involved solving the potential profile in the TFET and using that potential profile to calculate $G_{\text {TAT }}$ using the standard Schenk model. The $G_{\text {TAT }}$ was then approximated by the Gaussian distribution function for compact implementation. The model was compared against technology computer-aided design (TCAD) results and was found in reasonable agreement. The model was also used to fit an experimental device's $I_{\mathrm{ds}}-V_{\mathrm{gs}}$ characteristics. The results, while not exactly fitting the experimental data, follow the general experimental $I_{\mathrm{ds}}-V_{\mathrm{gs}}$ trend reasonably well; the subthreshold slope was loosely similar to the experimental device. Additionally, the ON-current, especially to make a high drain-source bias model accurate, can be further improved by including effects such as electrostatic degradation and series resistance.
\end{abstract}

Keywords: tunneling field-effect-transistor; trap-assisted-tunneling; Schenk model; line tunneling

\section{Introduction}

With conventional complementary metal-oxide-semiconductor (CMOS) technology coming to the end of its life cycle owing to scaling limitations, there has been significant interest in the research and development of alternate technologies including tunneling field-effect-transistors (TFET) [1-3]. TFETs work on the principle of band-to-band-tunneling, and provide a significantly better subthreshold slope (SS) as compared to metal-oxide-semiconductor field-effect-transistor (MOSFET) devices, and its ON-current $\left(I_{\mathrm{ON}}\right)$ issue [4] can be augmented with the help of alternate designs, including the line tunneling type TFET or III-V material TFET. In theory, this makes the TFET an ideal candidate to replace the aging MOSFET.

However, there is one issue that continues to be the bottleneck in the practical realization of the TFET. TFETs are very sensitive to subthreshold degradation caused by trap states [5]. Trap states cause trap-assisted-tunneling (TAT); TAT is a phonon-assisted band-to-band tunneling current generation process aided by trap states and is known to cause significant subthreshold slope degradation in TFETs. To date, there is very limited work available in the literature on the modeling of TAT mechanisms. Sajjad et al. [6] developed a compact TAT model based on Hurkx's TAT model [7]. However, Hurkx's TAT model can be considered a first-order model; it lacks fundamental and microscopic physical 
parameters that play a critical role in influencing TAT current including the phonon energy and Huang Rhys factor [8], which is a measure of the electron-phonon coupling and the lattice relaxation energy [9]. This makes fitting any experimental data with the help of the Hurkx model very difficult and without any physical relevance. Furthermore, the Hurkx model is a piece-wise model with different equations for different trap energy levels $\left(E_{\mathrm{T}}\right)$ [10]. This makes its compact implementation impractical. The Schenk model [11] on the other hand, offers a more microscopic approach to TAT modeling and is easy to implement numerically. The Schenk model has been used in studies investigating the role of TAT in TFET devices. However, there is no numerical or compact implementation of the Schenk model in the literature. This paper presents a simple Schenk-TAT compact model that is applied to fit the experimental drain-source current $\left(I_{\mathrm{ds}}\right)$-gate-source voltage $\left(V_{\mathrm{gs}}\right)$ data of a line tunneling type and L-shaped TFET (LTFET) device [12]. The model is discussed in Section 2, and the results are discussed in Section 3. The conclusion is presented in Section 4.

\section{Model Presentation}

Figure 1a shows the schematic of the LTFET [12]. The channel is sandwiched between the gate and source. The source with height $H_{\text {source }}=70 \mathrm{~nm}$ is heavily $\mathrm{p}^{+}\left(10^{19} \mathrm{~cm}^{-3}\right)$ doped with $H_{\text {source }}$ in the $y$ direction, and the channel is $\mathrm{n}^{-}\left(10^{15} \mathrm{~cm}^{-3}\right)$ doped. A part of the channel is also found underneath the source and does not overlap between the gate and source. This is termed as $R_{\text {nonoverlap. The part }}$ of the channel that overlaps between the source and gate is termed as $R_{\text {overlap }}$ and has a thickness $T_{\mathrm{j}}=10 \mathrm{~nm}$ in the $x$ direction and height $\left(H_{\text {overlap }}=H_{\text {source }}=70 \mathrm{~nm}[12,13]\right)$ in the $y$ direction. The $\mathrm{HfO}_{2}$ gate dielectric is $2 \mathrm{~nm}$ thick $\left(t_{\mathrm{ox}}\right)$. The drain is heavily $\mathrm{n}^{+}$doped $\left(10^{19} \mathrm{~cm}^{-3}\right)$.

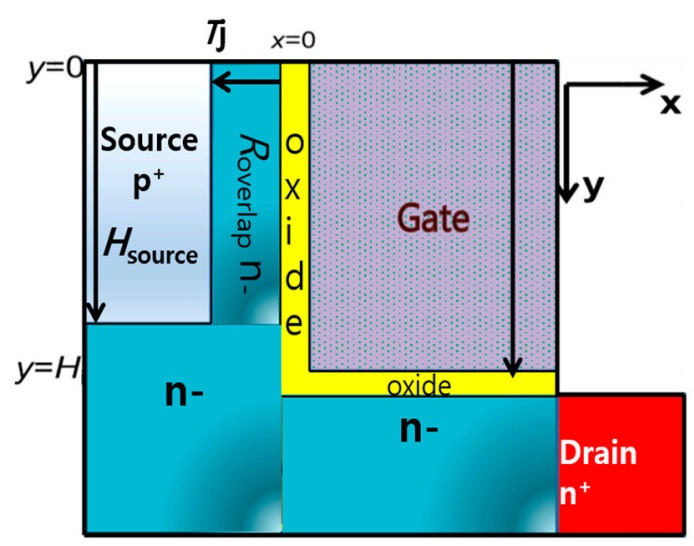

(a)

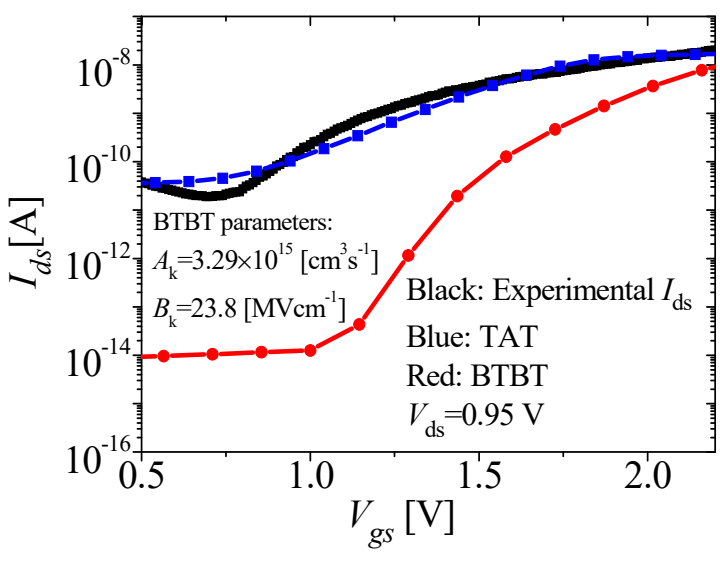

(b)

Figure 1. (a). Schematic of the L-shaped tunneling field-effect-transistor (LTFET). (b) Black: $I_{\mathrm{ds}}-V_{\mathrm{gs}}$ characteristics of experimental LTFET [12], blue: trap-assisted-tunneling (TAT) current calculated using the trap distribution and method in [13], and red: Band-to-band tunneling (BTBT) current fitted using dynamic nonlocal BTBT current [10].

Before delving into the details of the model, it is important to illustrate the different current mechanisms in line tunneling TFETs. There are three different current mechanisms including (1) 2D BTBT, (2) 1D BTBT, and (3) TAT. 2D BTBTs originate from the corner-effect present in line tunneling TFETs. Because of the corner shape present in line tunneling TFETs, where the source and channel meet, the electric field converges around the sharp source corner, and increases the potential in the area surrounding the corner region [14]. This increased potential causes a 2D BTBT. The threshold voltage of 2D BTBT is lower than that of a 1D BTBT. However, the threshold voltage of the TAT is lower than that of the 2D BTBT and, as a consequence, the TAT current is generated at a lower $V_{\text {gs }}$ than both the 2D and 1D BTBT currents. This is summarized in Table 1. Details of the 1D and 2D BTBT mechanisms can be found in $[15,16]$. Depending on the number of traps, their energy level, density and whether the traps 
are bulk or interface traps, the TAT generated drain current can vary. In most cases, the TAT current dominates the low $V_{\text {gs }}$ bias subthreshold-BTBT current of TFETs [5,6], but in some cases such as the one reported in [13], the TAT current can dominate over the BTBT current for the entire $V_{\text {gs }}$ range. This is explained with the help of Figure $1 \mathrm{~b}$. Figure $1 \mathrm{~b}$ shows the $I_{\mathrm{ds}}-V_{\mathrm{gs}}$ characteristics of the experimental LTFET [12]. It was first fitted using a dynamic nonlocal BTBT model [10] with theoretically determined, material-dependent $A_{\mathrm{k}}=3.9 \times 10^{15} \mathrm{~cm}^{3} / \mathrm{s}$, and $B_{\mathrm{k}}=23.8 \mathrm{MV} / \mathrm{cm}$ parameters. However, the BTBT- $I_{\mathrm{ds}}$ was found to be too low. With the BTBT current failing to match the experimental device's $I_{\mathrm{ds}}$, it was determined that it is instead the TAT-generated current that dominates for the entire $V_{\mathrm{gs}}$ range [13], as shown in Figure $1 b$.

Table 1. Current mechanisms in line tunneling TFETs and their threshold voltages.

\begin{tabular}{clc}
\hline Mechanism & \multicolumn{1}{c}{ Region Dominated } & Threshold Voltage \\
\cline { 1 - 2 } 2D BTBT & Typically intermediate bias region. & \multirow{2}{*}{ TAT < 2D BTBT < 1D BTBT } \\
\cline { 1 - 2 } 1D BTBT & Typically subthreshold region. & $\begin{array}{l}\text { Typically subthreshold and intermediate bias regions [5], } \\
\text { and in extreme cases [13] the high bias region as well. }\end{array}$ \\
\hline TAT & &
\end{tabular}

For simplicity, only the 1D potential model is considered [15], and any 2D effects [16] are ignored. The model is divided into two parts. The first part deals with the potential model which has already been reported in detail in [16] and is only briefly described here. Next is the standard Schenk model itself, which has been taken from [10] followed by its compact implementation using the Gaussian distribution function.

\subsection{Potential Model}

Figure 2a shows the TAT generation rate $\left(G_{\mathrm{TAT}}\right)$ contour plot at $V_{\mathrm{gs}}=0 \mathrm{~V}$ and $V_{\mathrm{ds}}=0.5 \mathrm{~V}$ for an LTFET with $T_{\mathrm{j}}=10 \mathrm{~nm}$. Figure $2 \mathrm{~b}$ shows the $G_{\mathrm{TAT}}$ extracted from the contour plot along the cutline indicated in Figure 2a, along with $G_{\mathrm{TAT}}$ at several $V_{\mathrm{gs}}$ biases ranging from 0 to $2 \mathrm{~V}$. The region shown along the cutline is the reference region of the model, where all parameters including potential and $G_{\mathrm{TAT}}$ are calculated and assumed to be constant in the $y$ and $z$ directions. It can be seen that the $G_{\mathrm{TAT}}$ is significant only in the channel region, and for that reason, this model calculates $G_{\text {TAT }}$ only in the channel region.

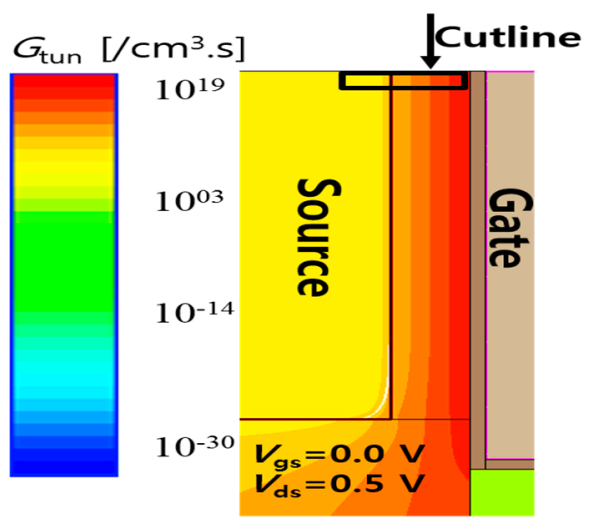

(a)

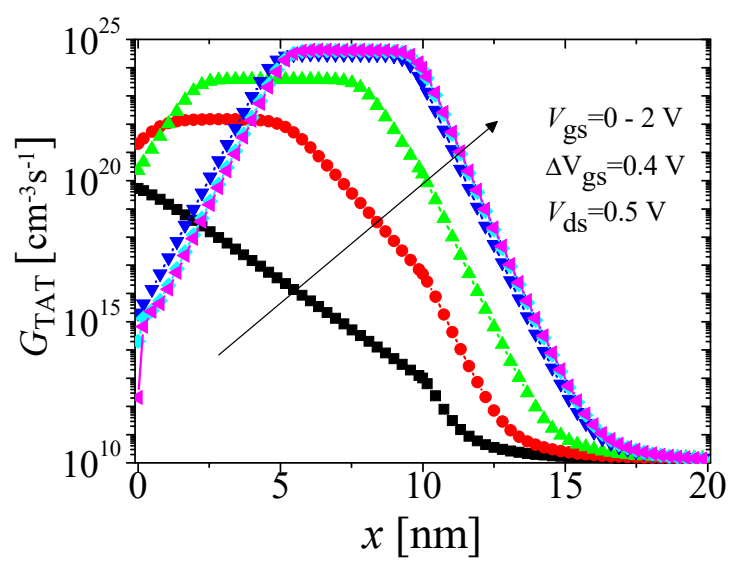

(b)

Figure 2. Cont. 


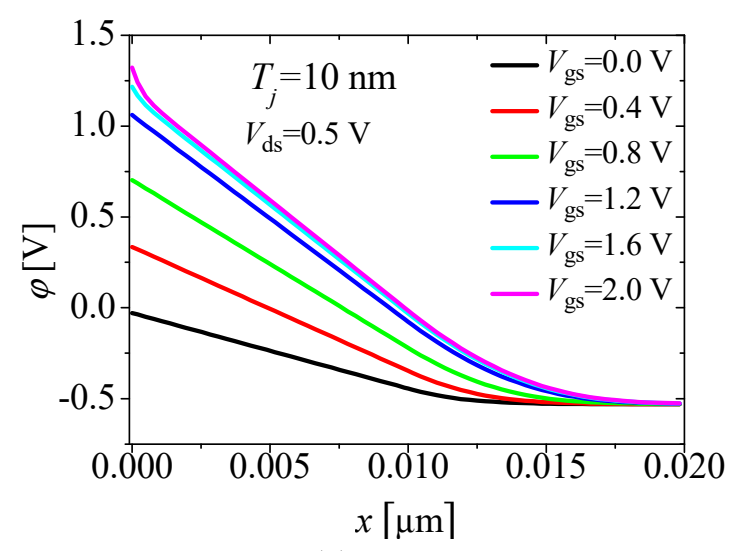

(c)

Figure 2. (a) $G_{\mathrm{TAT}}$ contour plot at $V_{\mathrm{gs}}=0$ and $V_{\mathrm{ds}}=0.5 \mathrm{~V}$ for an LTFET with $T_{\mathrm{j}}=10 \mathrm{~nm}$. (b) $G_{\mathrm{TAT}}$ extracted along the cutline in Figure 2a, for $V_{\mathrm{gs}}=0-2 \mathrm{~V}$ with $\Delta V_{\mathrm{gs}}=0.4 \mathrm{~V}$. (c) Potential along the cutline of Figure $2 \mathrm{a}$ at different $V_{\mathrm{gs}} \mathrm{s}$ for $V_{\mathrm{ds}}=0.5 \mathrm{~V}$ and $T_{\mathrm{j}}=10 \mathrm{~nm}$.

The potential model is based on solving the Poisson equation twice in the source and $R_{\text {overlap }}$ regions. As a result, the potential and electric field $(E)$ equations in the respective regions are given below [16]. Table 2 mentions the meaning of each symbol used in the following equations:

$$
\begin{gathered}
\frac{\partial \varphi_{\text {source }}}{\partial x_{\text {source }}}=\frac{q N_{a}}{\varepsilon_{s i}}\left(x_{\text {source }}-L_{\text {dep }}\right) \\
\frac{\partial \varphi_{\text {channel }}}{\partial x_{\text {channel }}}=-\frac{q N_{d}}{\varepsilon_{s i}} x_{\text {channel }}-\frac{\varepsilon_{o x}}{\varepsilon_{s i} t_{o x}}\left(V_{g s}-V_{f b}-\varphi_{s}\right) \\
\varphi_{\text {source }}\left(x_{\text {source }}\right)=\frac{q N_{a}}{2 \varepsilon_{s i}}\left(x_{\text {source }}-L_{d e p}\right)^{2}+\varphi_{\text {dep }} \\
\varphi_{\text {channel }}\left(x_{\text {channel }}\right)=-\frac{q N_{d}}{2 \varepsilon_{s i}} x_{\text {channel }}^{2}-\frac{\varepsilon_{\text {ox }}}{\varepsilon_{s i} t_{\text {ox }}}\left(V_{g s}-V_{f b}-\varphi_{s}\right) x_{\text {channel }}+\varphi_{s} \\
\varphi_{s}=V_{g s}-V_{f b}+\frac{q\left(N_{a}+N_{d}\right)}{C_{o x}} T_{j}+\frac{q \varepsilon_{s i} N_{a}}{C_{o x}^{2}} \\
-\gamma \sqrt{V_{g s}-V_{f b}+\frac{q\left(N_{a}+N_{d}\right)}{2 \varepsilon_{s i}} T_{j}^{2}+\frac{q\left(N_{a}+N_{d}\right)}{C_{o x}} T_{j}+\frac{q \varepsilon_{s i} N_{a}}{2 C_{o x}^{2}}-\varphi_{\text {dep }}}
\end{gathered}
$$

where $\gamma=\sqrt{{ }_{s i} q N_{d}} / C_{o x}$. The potential profile within the channel is assumed to be linear.

$$
\varphi_{\text {channel }}\left(x_{\text {channel }}\right)=m x_{\text {channel }}+\varphi_{s}
$$

where $m$ is the slope of the linear potential profile in the channel, $m=\left(\varphi_{s}-\varphi_{j}\right) / T_{j}$, or the electric field. Equation (6) is not derived from (2). It is a convenient assumption for compact modeling purposes. It can be seen in Figure $2 c$ that potential follows a linear potential profile within the channel. Due to the strong band-bending, this assumption quasi-breaks down at higher $V_{g s}$ bias. This consequence is explained later in Section 3. Because of the linear potential profile, $E$ is constant throughout the channel and $\varphi_{j}$ can be derived as

$$
\varphi_{j}=\varphi_{s}+\frac{q T_{j}^{2}\left(N_{d}+2 N_{a}\right)}{2 \varepsilon_{s i}}-\sqrt{\frac{\left(2 q N_{a} T_{j}^{2}\right)\left(\varphi_{s}+V_{b i s}\right)}{\varepsilon_{s i}}+\frac{q^{2} T_{j}^{4} \cdot\left(N_{d} N_{a}+N_{a}^{2}\right)}{\varepsilon_{s i}^{2}}}
$$


Table 2. List of symbols specifically used in (1)-(7).

\begin{tabular}{cll}
\hline Symbol & \multicolumn{1}{c}{ Description } & \multicolumn{1}{c}{ Value/Unit } \\
\hline$\varphi_{\text {source }}$ & Potential at source edge & $\mathrm{V}$ \\
\hline$N_{\mathrm{a}}, N_{\mathrm{d}}$ & Source, channel doping & $\mathrm{cm}^{-3}$ \\
\hline$x_{\text {source }}, x_{\text {channel }}$ & $x$ co-ordinate in source, channel & $\mathrm{cm}$ \\
\hline$L_{\mathrm{dep}}$ & Depletion length in source & $\mathrm{cm}$ \\
\hline$\varepsilon_{s i,}, \varepsilon_{\text {ox }}$ & Silicon, oxide permittivity & $11.9_{\varepsilon 0}, 25_{\varepsilon 0}$ \\
\hline$V_{\mathrm{fb},}$ & Flat band voltage & $\mathrm{V}$ \\
\hline$q$ & Electron charge & $1.6 \times 10^{-19} \mathrm{C}$ \\
\hline$\varphi_{\mathrm{s},} \varphi_{\mathrm{j},}, \varphi_{\mathrm{channel}}$ & Surface potential at $x_{\text {channel }}=0$, junction potential at $x=$ & $\mathrm{V}$ \\
\hline$\varphi_{\text {;dep }}$ & $T_{\mathrm{j}}$, and potential as a function of $x_{\text {channel }}$, respectively & \\
\hline$C_{\mathrm{ox}}$ & Depletion potential in source & $\mathrm{V}$ \\
\hline$V_{\mathrm{bis}}$ & Gate capacitance & $\mathrm{F} / \mathrm{cm}^{2}$ \\
\hline
\end{tabular}

\subsection{Schenk Model}

The Schenk model $[7,10]$ works by modifying the lifetime parameter $\left(\tau_{0}\right)$ in the original Shockley-Read-Hall (SRH) recombination-generation model through the $\Gamma$ parameter as follows:

$$
\tau_{n(p)}=\frac{\tau_{0 n(p)}}{1+\Gamma_{n(p)}(E)}
$$

where $n(p)$ in (8) represent electrons and holes, respectively. The $\Gamma$ accounts for the $E$-driven, and phonon-assisted TAT process, and is calculated by the Schenk model. If there is any TAT present, $\Gamma$ is usually significantly higher than 1 [5]. Otherwise, it is zero. The lifetime given by (8) features in the SRH model expression, which is used to give $\mathrm{G}_{\mathrm{TAT}}$ if $\Gamma>0$, as follows:

$$
G_{T A T}=\frac{n p-n_{i}^{2}}{\frac{\tau_{0 p}}{1+\Gamma_{p}}\left(n+n_{1}\right)+\frac{\tau_{0 n}}{1+\Gamma_{n}}\left(p+p_{1}\right)}
$$

where $n_{\mathrm{i}}$ is an intrinsic carrier concentration, $n_{1}=n_{i} \exp \left(E_{T} / k T\right), p_{1}=n_{i} \exp \left(E_{T} / k T\right)$, and $k$ and $T$ are the Boltzmann constant and temperature, respectively. $n$ and $p$ in (9) are given by

$$
n=n_{i} \cdot \exp \left(\frac{\varphi(x)-\varphi_{\text {equasi }}}{v_{\text {th }}}\right), p=n_{i} \cdot \exp \left(-\frac{\varphi(x)}{v_{\text {th }}}\right)
$$

where $v_{\text {th }}$ and $\varphi_{\text {equasi }}$ present thermal voltage and electron quasi Fermi level, respectively. Substituting for $\varphi_{\text {channel }}$ from (6) in (10), $n$ and $p$ are given by

$$
n(x)=n_{i} \cdot \exp \left(\frac{m x+\varphi_{s}-\varphi_{\text {quasi }}}{v_{t h}}\right), p(x)=n_{i} \cdot \exp \left(-\frac{m x+\varphi_{s}}{v_{t h}}\right) .
$$

$\Gamma$ is calculated by the following equation. A detailed derivation of this equation is given in [7].

$$
\begin{gathered}
\Gamma_{n(p)}=\left(1+\frac{\left(h \Theta_{n(p)}\right)^{\frac{3}{2}} \sqrt{E_{\operatorname{tn}(p)}-E_{0 n}(p)}}{E_{0 n(p)} h \omega_{0}}\right)^{-\frac{1}{2}} \frac{\left(h \Theta_{n(p)}\right)^{\frac{3}{4}}\left(E_{t n(p)}-E_{0 n(p)}\right)^{\frac{1}{4}}}{2 \sqrt{E_{t n(p)} E_{0 n(p)}}}\left(\frac{h \Theta_{n(p)}}{k T}\right)^{\frac{3}{2}} . \\
\exp \left(-\frac{E_{t n(p)}-E_{0 n(p)}}{h \omega_{0}}+\frac{h \omega_{0}-k T}{2 h \omega_{0}}+\frac{2 E_{t n(p)}+k T}{2 h \omega_{0}} \ln \frac{E_{t n(p)}}{\varepsilon_{R}}-\frac{E_{0 n(p)}}{h \omega_{0}} \ln \frac{E_{0 n(p)}}{\varepsilon_{R}}+\frac{E_{t n(p)}-E_{0 n(p)}}{k T}-\frac{4}{3}\left(\frac{E_{t n(p)}-E_{0 n(p)}}{h \Theta_{n(p)}}\right)^{\frac{3}{2}}\right) .
\end{gathered}
$$


Here, $\hbar$ is the reduced Planck's constant, $\hbar \omega_{0}$ is the effective phonon energy $\left(E_{\text {phon }}\right), \varepsilon_{\mathrm{R}}=S E_{\text {phon }}$ is the lattice relaxation energy [17] which is known to influence the subthreshold slope (SS), where $S$ is the Huang-Rhys factor [18] which is a measure of electron-phonon coupling. Both $E_{\text {phon }}$ and $S$ are known to influence $\Gamma$, although the effect of $E_{\text {phon }}$ has been found to be dominant over $S$ on $\Gamma$. Furthermore, the current can change if either $S$ or $E_{\text {phon }}$ change, even if $\varepsilon_{R}$ remains constant [18]. $E_{\operatorname{tn}(p)}$ is the energy at which the tunneling probability for the carriers to the tunnel from $E_{\mathrm{T}}$ to the band edge is the maximum and $\Theta_{n(p)}=\left(q^{2} E^{2} / 2 \hbar m_{n(p)}\right)^{1 / 3}$ is the electro-optical frequency [18]. $m_{n}$ and $m_{p}$ are the electron and hole tunneling masses, respectively.

The model parameters including trap level $E_{\mathrm{T}}, S, E_{\text {phon, }}$ and ${ }_{n(p)}$ are the fundamental quantities that strongly influence the TAT process. The first three can be determined experimentally using Deep Level Transient Spectroscopy $[19,20]$. The electro-optical frequency is dependent on the electric field and the tunneling masses. This restricts the model to being a local TAT model [18]. $E_{0 n(p)}$ is the energy of an optimum horizontal transition path which is dependent on the field strength and temperature. This determines the most probable recombination path [18], and is given by

$$
E_{0 n(p)}=2 \sqrt{\varepsilon_{F n(p)}}\left[\sqrt{\varepsilon_{F n(p)}+E_{t n(p)}+\varepsilon_{R}}-\sqrt{\varepsilon_{F n(p)}}\right]-\varepsilon_{R}
$$

where $\varepsilon_{F n(p)}=\frac{\left(2 \varepsilon_{F} k T\right)^{2}}{\left(h \Theta_{n(p)}\right)^{3}} . E_{\mathrm{tn}}$ for electrons is given by

$$
E_{t n}=\frac{1}{2} E_{b g n}+\frac{3}{4} k T \ln \left(\frac{m_{n}}{m_{p}}\right)-E_{T}-\left(32 R_{c} h^{3} \Theta^{3}\right)^{\frac{1}{4}}
$$

and $E_{\mathrm{tp}}$ is given by

$$
E_{t p}=\frac{1}{2} E_{b g n}+\frac{3}{4} k T \ln \left(\frac{m_{n}}{m_{p}}\right)-E_{T}+\left(32 R_{v} h^{3} \Theta^{3}\right)^{\frac{1}{4}}
$$

where $R_{\mathrm{c}(\mathrm{v})}$ are the effective Rydberg energies; $R_{\mathrm{c}(\mathrm{v})}=m_{\mathrm{n}(\mathrm{p})} R_{\mathrm{y}} / \varepsilon^{2}$ for electrons (holes), where $R_{\mathrm{y}}$ $(=13.6 \mathrm{eV})$ is the Rydberg energy and $\varepsilon$ is the permittivity.

\subsection{Compact Implementation}

For compact implementation, $G_{\mathrm{TAT}}$ given by (9) is approximated using a Gaussian distribution function as follows:

$$
G_{T A T}=G_{T A T \_\max } \exp \left(-\left(\frac{x-x_{\max }}{x_{\text {width }}}\right)^{2}\right)
$$

where $x_{\text {width }}$ is the width of the Gaussian distribution. $G_{\text {TAT_max }}$ is the maximum $G_{\text {TAT }}$ along the channel region, and $x_{\max }$ is the $x$-point where $G_{\text {TAT }}$ is maximum. $\mathrm{G}_{\text {TAT_max }}$ always occurs where $n=p$. This can be seen in Figure 3 which shows $n, p$ (left axis), and $G_{\mathrm{TAT}}$ (right axis) at different $V_{\mathrm{gs}} \mathrm{s}$ for $V_{\mathrm{ds}}=0.5 \mathrm{~V}$, for an LTFET with $T_{\mathrm{j}}=10 \mathrm{~nm}$. It can be seen from Figure 3 that at the point where $n=p$, $G_{\mathrm{TAT}}$ is maximum. The $x$ co-ordinate where this occurs is labelled as $\mathrm{x}_{\max } \cdot \mathrm{x}_{\max }$ can be found by equating $n=p$, as follows: and solving for $x$, that is.

$$
\begin{gathered}
n_{i} \exp \left(\frac{m x_{\text {max }}+\varphi_{s}-\varphi_{\text {equasi }}}{v_{\text {th }}}\right)=n_{i} \exp \left(-\frac{m x_{\text {max }}+\varphi_{s}}{v_{\text {th }}}\right) \\
x_{\text {max }}=\frac{\varphi_{\text {equasi }}-\left(2 \varphi_{s}\right)}{2 m}
\end{gathered}
$$

$G_{\text {TAT_max }}$ is found by using $x_{\max }$ in (9), as follows:

$$
G_{T A T_{-} \max }=\frac{n\left(x_{\max }\right) p\left(x_{\max }\right)-n_{i}^{2}}{\frac{\tau_{0 p}}{1+\Gamma_{p}}\left[n\left(x_{\max }\right)+n_{1}\right]+\frac{\tau_{0 n}}{1+\Gamma_{n}}\left[p\left(x_{\max }\right)+p_{1}\right]}
$$


where $n\left(x_{\max }\right)$ and $p\left(x_{\max }\right)$ can be found using $x=x_{\max }$ in (11). It should be mentioned that $\Gamma$ is independent of $x$; constant electric field in the channel makes $\Gamma$ calculated from (12) essentially independent of $x$. In order to find $x_{\text {width }}$, the standard deviation $(\sigma)$ of the Gaussian distribution is utilized. $\sigma$ is obtained using standard equations [21]. Utilizing the Empirical Rule in Statistics and Probability [21] which states that $99.7 \%$ of values in a normal distribution lie within 3 standard deviations of the mean value [21], the width of Gaussian distribution was approximated at 3 standard deviations of the maximum value of the function, that is, the width was estimated to be the $x$ co-ordinate ( $x_{\text {width }}$ ) that satisfied this condition, $3 \sigma$ of $G_{T A T}$, symbolized by $G_{T A T \_3 \sigma}$. Substituting this condition in (9), along with (11), assuming mid-gap trap level so that $n_{1}=p_{1}=n_{i},(9)$ can be given by

$$
G_{T A T_{\_} 3 \sigma}=\frac{n_{i}^{2} \exp \left(\frac{-\varphi_{\text {quasi }}}{v_{\text {th }}}\right)-n_{i}^{2}}{\frac{\tau_{0 p}}{1+\Gamma_{p}}\left(n+n_{i}\right)+\frac{\tau_{0 n}}{1+\Gamma_{n}}\left(p+n_{i}\right)}
$$

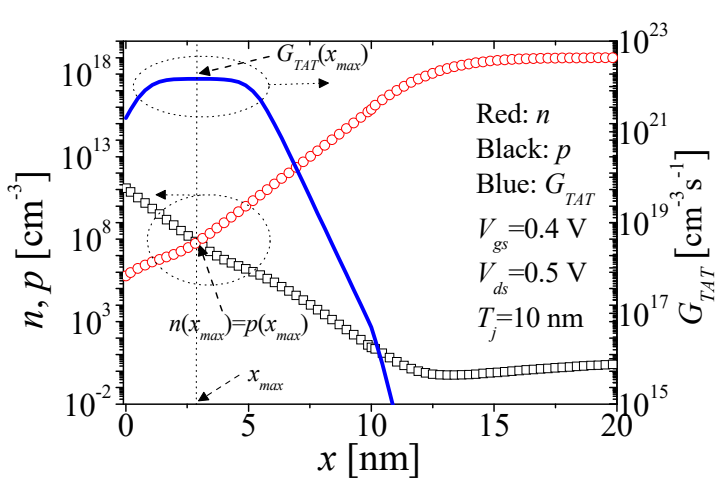

(a)

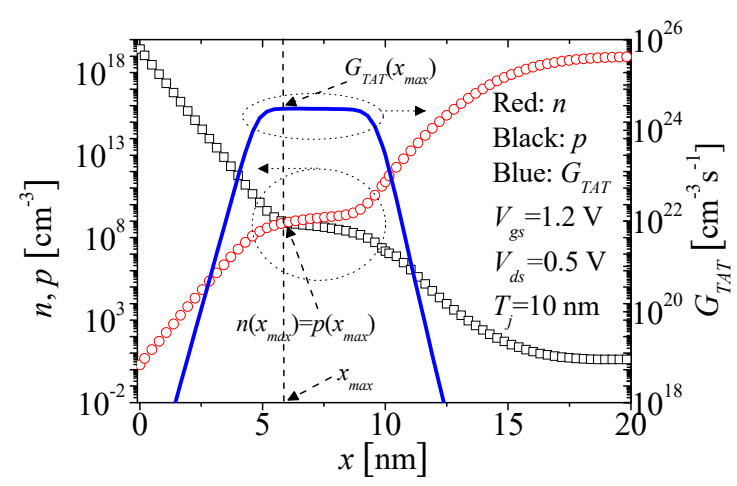

(c)

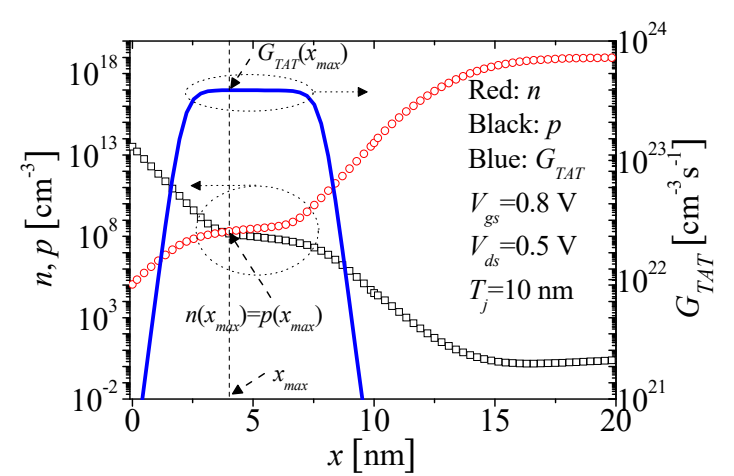

(b)

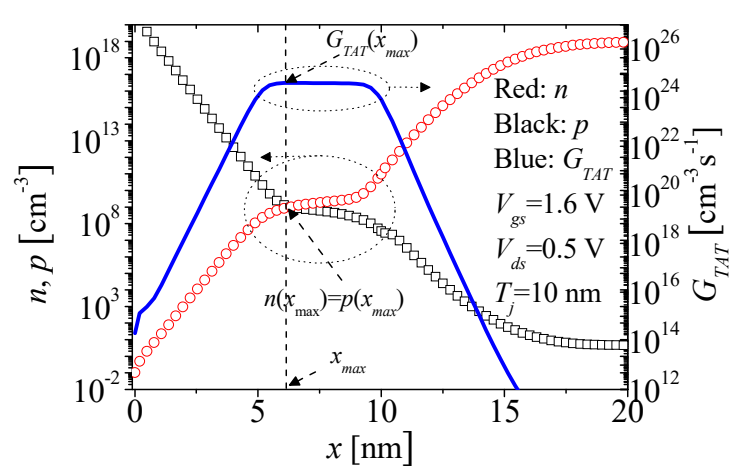

(d)

Figure 3. (a-d) $n, p$ (left-axis), and $G_{\mathrm{TAT}}$ (right-axis) at $V_{\mathrm{gs}}=0.4,0.8,1.2$, and $1.6 \mathrm{~V}$, respectively, all at $V_{\mathrm{ds}}=0.5 \mathrm{~V}$. The location where $n=p$, and of $G_{\mathrm{TAT} \_ \text {Max }}$, that is at $x=x_{\max }$ are identified by arrows.

It can be seen from Figures 3 and 4 that at any bias, the width of the Gaussian distribution $n>>p$. Furthermore, assuming equivalent $\tau_{0 \mathrm{n}}=\tau_{0 \mathrm{p}}=\tau_{0}$, and $\Gamma_{\mathrm{n}} \sim \Gamma_{\mathrm{p}}$ which is true for the materials with $m_{\mathrm{e}} \sim m_{\mathrm{p}}$ including silicon, and advanced III-V InGaAs-based materials [13], (20) can be expressed as

$$
G_{T A T_{-} 3 \sigma}=\frac{n_{i}^{2} \exp \left(\frac{-\varphi_{\text {quasi }}}{v_{\text {th }}}\right)-n_{i}^{2}}{\frac{\tau_{0}}{1+\Gamma_{n}}\left[n_{i}\left(\exp \left(\frac{m x_{\text {width }}+\varphi_{s}-\varphi_{\text {quasi }}}{v_{\text {th }}}\right)+2\right)\right]}
$$


Taking $\log$ on both sides, ignoring 2 , and solving for $x_{\text {width }}, x_{\text {width }}$ can be given by

$$
x_{\text {width }}=\frac{\left\{\log \left[\left(G_{T A T_{-} 3 \sigma}\right)\left(\frac{\tau_{0}}{1+\Gamma_{n}}\right)\right]-\log \left(n_{i}\right)\right\} \cdot\left(v_{t h}-\varphi_{s}\right)}{m}
$$

The TAT drain current $\left(I_{\mathrm{ds} \_ \text {TAT }}\right)$ is given by the following expression:

$$
\begin{aligned}
& I_{d s_{-} T A T}=q \int_{0}^{a b s\left(T_{j}\right)} \int_{0}^{H_{\text {source }}} \int_{0}^{W} G_{T A T} d x d y d z=q W H_{\text {source }} \int_{0}^{a b s\left(T_{j}\right)} G_{T A T} d x \\
& I_{d s_{-} T A T}=\sqrt{\pi} q W H_{\text {source }} G_{T A T_{-} M a x} x_{\text {width }}\left[\operatorname{erf}\left(\frac{a b s\left(T_{j}\right)-x_{\max }}{x_{\text {width }}}\right)-\operatorname{erf}\left(\frac{0-x_{\text {max }}}{x_{\text {width }}}\right)\right]
\end{aligned}
$$

where $W$ is the width of the device. It is assumed that $G_{\mathrm{TAT}}$ is independent of $H_{\text {source, }}$ and $W$. Note that (24) is simply the closed-integral expression of (16) integrated between $T_{\mathrm{j}}$ and $0 \mu \mathrm{m}$ at the surface.

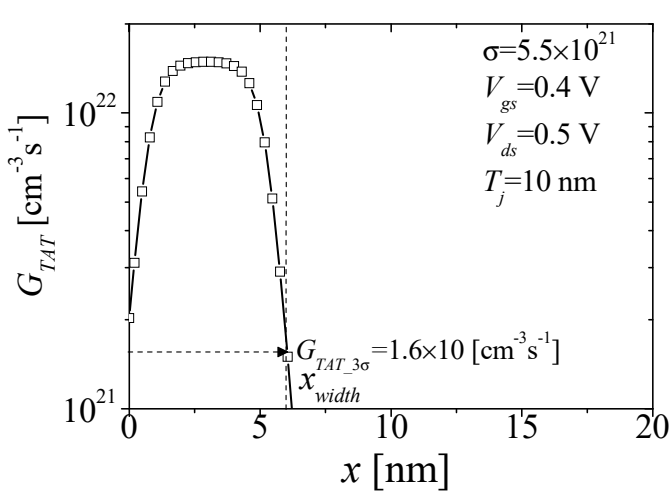

(a)

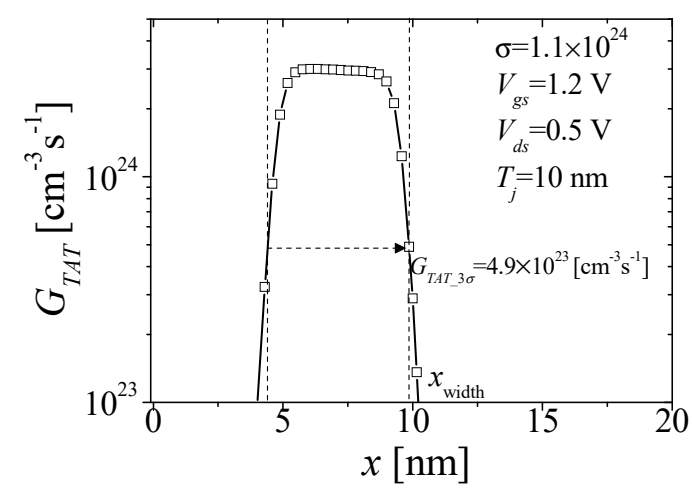

(c)

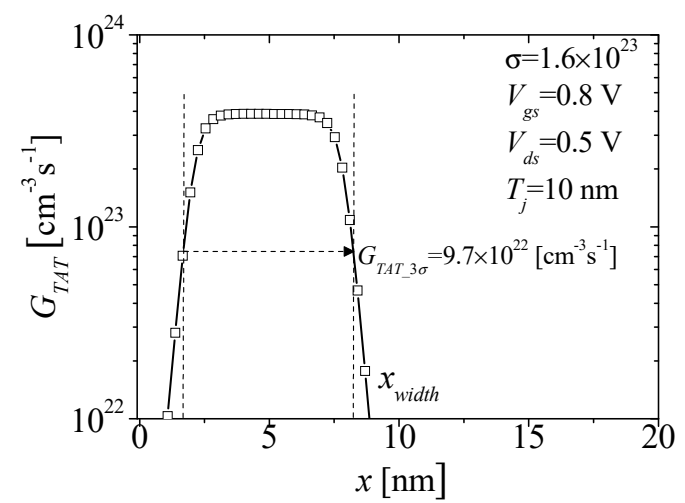

(b)

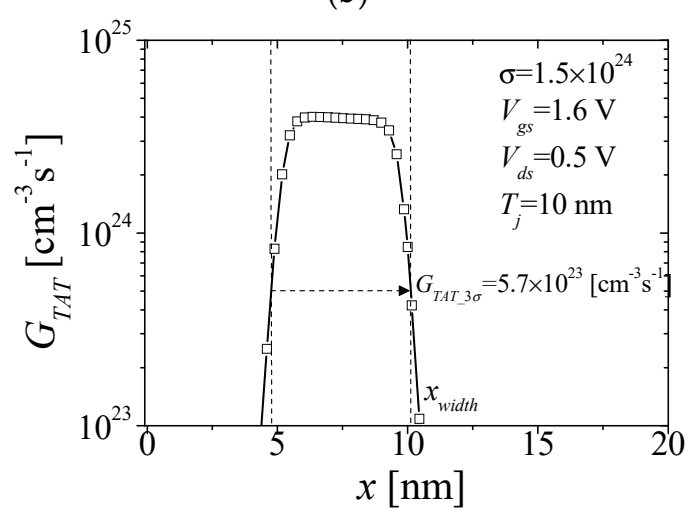

(d)

Figure 4. (a-d) Illustration of the method used to estimate the width of the Gaussian distribution. $G_{\mathrm{TAT}}$ is shown, and $x_{\text {width }}$ is pointed out by arrows, for $V_{\mathrm{gs}}=0.4,0.8,1.2$, and $1.6 \mathrm{~V}$, respectively.

\section{Results}

To demonstrate whether the Gaussian approximation of $G_{\mathrm{TAT}}$ is feasible, Figure 5 compares $G_{\text {TAT }}$ calculated from the actual SRH-based $G_{\text {TAT }}$ equation: (9) and $G_{\text {TAT }}$ approximated by Gaussian approximation: (16) for $T_{j}=7 \mathrm{~nm}$. The approximated $G_{\mathrm{TAT}}$ is reasonably consistent with the calculated $G_{\text {TAT }}$. It should be emphasized that this is only an approximation for compact modeling purposes. The approximated $G_{\text {TAT }}$ overestimates the actual $G_{\text {TAT }}$, particularly at high bias values. The overestimation at higher $V_{\mathrm{gs}}$ bias is because the linear potential profile assumption does not remain valid at high $V_{\mathrm{gs}}$ bias. Figure $5 \mathrm{~b}$ shows two integrated $G_{\mathrm{TAT}}$. Based on Figure $5 \mathrm{a}$, b, some overestimation of $I_{\text {ds__TAT }}$ can be expected at high $V_{\text {gs }}$ bias. 


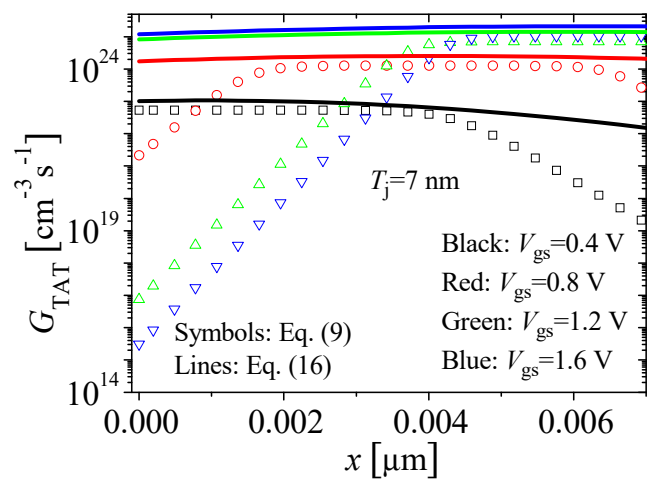

(a)

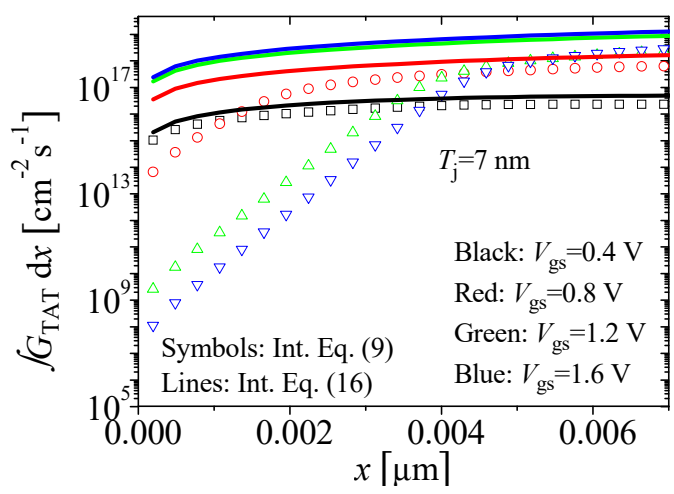

(b)

Figure 5. (a) $G_{\mathrm{TAT}}$ calculated from Equation (9) (symbols) compared against $G_{\mathrm{TAT}}$ calculated from Equation (16) (lines) for $V_{\mathrm{gs}}=0.4,0.8,1.6$, and $2.0 \mathrm{~V}$ shown by black, red, green and blue colors respectively, for $V_{\mathrm{ds}}=0.5 \mathrm{~V}$ and $T_{\mathrm{j}}=7 \mathrm{~nm}$. (b) Integrated $G_{\mathrm{TAT}}, \int_{T_{j}}^{0} G_{T A T} d x$ shown in Figure 5a. Same annotation as Figure 5a except symbols: Integral of Equation (9), and lines: Integral of Equation (16).

Figure 6 compares $I_{\text {ds_TAT }}$ from the technology computer-aided design (TCAD) (symbol) against $I_{\mathrm{ds} \text { _TAT }}$ calculated from (24) for different $T_{\mathrm{j}}$. Considering that this is a compact model, a reasonable agreement on the simulation results between the TCAD and the model is observed. The expected overestimation in $I_{\mathrm{ds} \_\mathrm{TAT}}$ at high $V_{\mathrm{gs}}$ bias can be seen in Figure 6. Figure 7 compares $I_{\mathrm{ds} \_\mathrm{TAT}}$ from the TCAD (symbols) against $I_{\mathrm{ds} \text { _TAT }}$ calculated from the model for $T_{\mathrm{j}}=10 \mathrm{~nm}$ and different $H_{\text {source, }}$ at $V_{\mathrm{ds}}$ $=0.5 \mathrm{~V}$. The slight disagreement at higher bias is due to the linear potential approximation which is not very accurate at higher bias due to strong surface band bending.

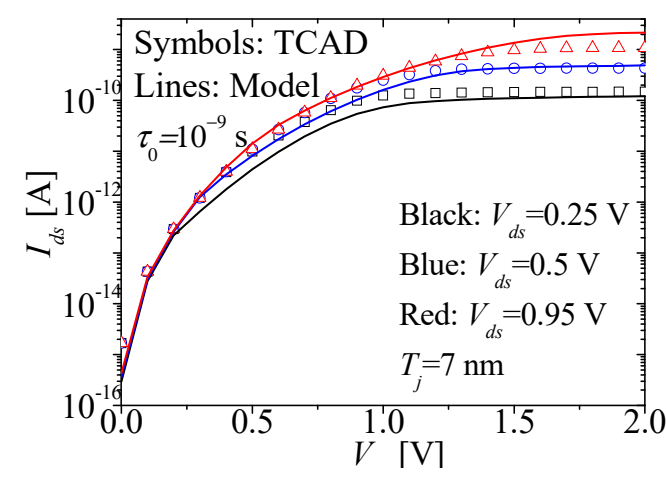

(a)

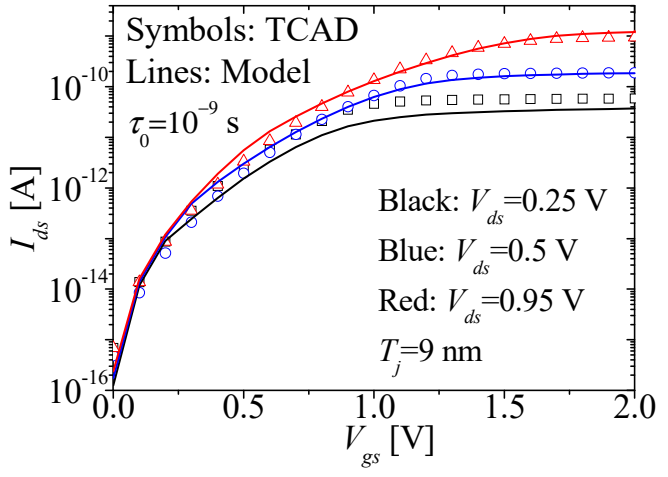

(c)

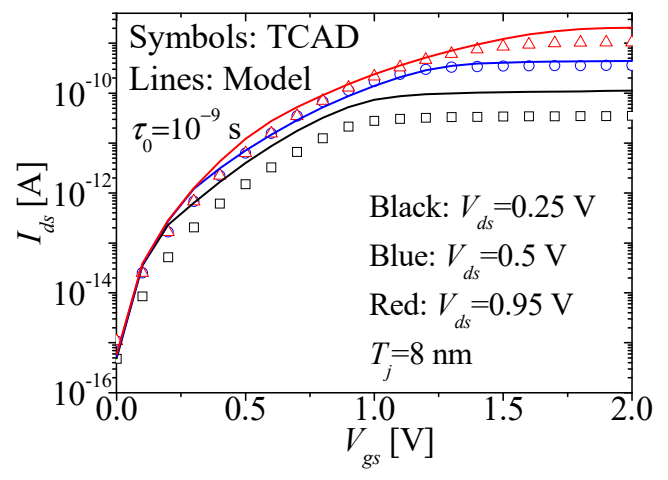

(b)

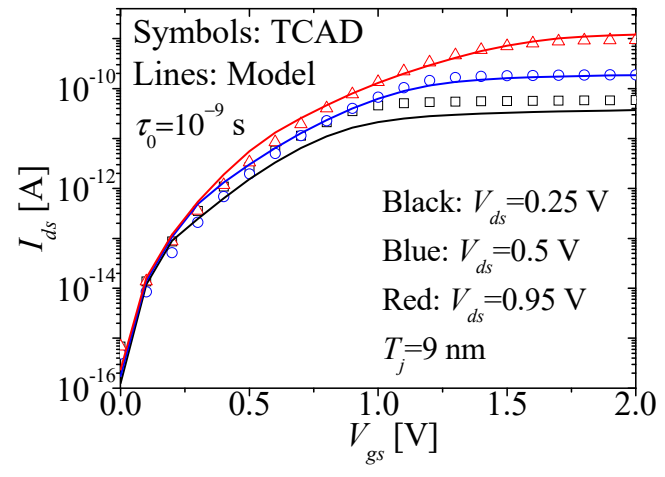

(d)

Figure 6. $I_{\mathrm{ds} \_ \text {TAT }}$ from technology computer-aided design (TCAD) (symbols) compared with $I_{\text {ds_TAT }}$ calculated from (24) for $T_{\mathrm{j}}=7 \mathrm{~nm}(\mathbf{a}), 8 \mathrm{~nm}(\mathbf{b}), 9 \mathrm{~nm}(\mathbf{c})$ and $10 \mathrm{~nm}(\mathbf{d})$. Black, blue, and red represent $V_{\mathrm{ds}}=0.25,0.5$, and $0.95 \mathrm{~V}$, respectively. 


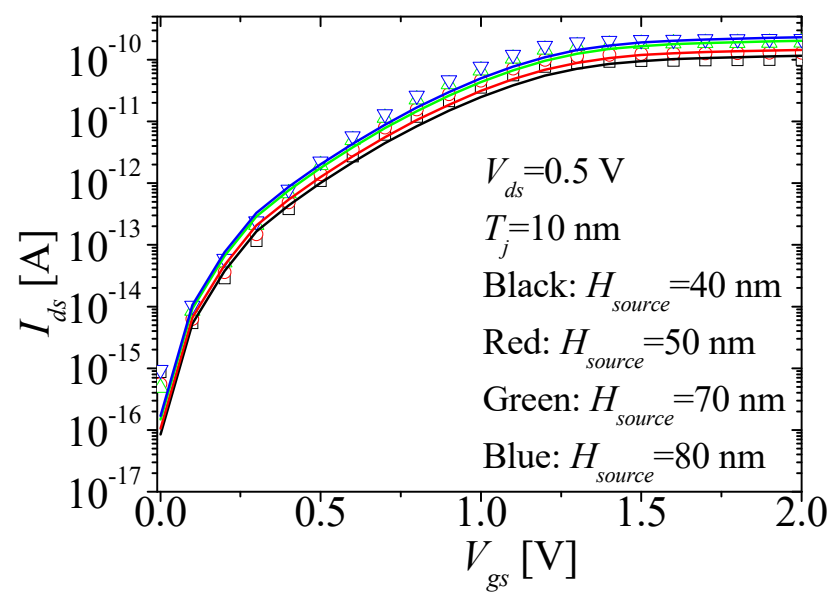

Figure 7. $I_{\mathrm{ds}}$ from TCAD (symbols) versus $I_{\mathrm{ds}}$ calculated by the model for an LTFET with $T_{\mathrm{j}}=10 \mathrm{~nm}$, and different $H_{\text {source }}=40 \mathrm{~nm}$ (black), $50 \mathrm{~nm}$ (red), $70 \mathrm{~nm}$ (green) and $80 \mathrm{~nm}$ (blue) at $V_{\mathrm{ds}}=0.5 \mathrm{~V}$.

To truly test the relevancy of the model, the model was used to fit experimental data from [12]. It was found in [13] that $I_{\mathrm{ds} \_ \text {TAT }}$ dominates the entire $V_{\mathrm{gs}}$ range for all $V_{\mathrm{ds}}$ biases. Therefore, $I_{\mathrm{ds} \_ \text {TAT }}$ from the model was compared against the experimental data. The result is shown in Figure 8. The model was calibrated to match the experimental $I_{\mathrm{ds}}-V_{\mathrm{gs}}$ characteristics at $V_{\mathrm{ds}}=0.95 \mathrm{~V}$ by choosing $\tau_{0}=10^{-11} \mathrm{~s}$. The model follows the experimental $\mathrm{I}_{\mathrm{ds}}$ trend very well. However, it is not entirely accurate, especially at low $V_{\mathrm{ds}}$. The reason for this is that (1) the electrostatic degradation caused by the trap states was not considered in this work. Electrostatic degradation causes the potential to degrade, and this effect becomes more important at high bias. To account for that, the Poisson and trap charge need to be calculated self-consistently. Furthermore, a different potential profile expression other than the linear potential profile given by (6) will be needed. This is because with the traps causing electrostatic degradation, the degraded potential profile changes from being linear to non-linear. It should be emphasized that from Figure 1b, BTBT current calculated using experimentally determined $A_{\mathrm{k}} / B_{\mathrm{k}}$ parameters was found to be too low than the current of the experimental device. The absence of BTBT current in this particular example is not the result of mismatch. An ambipolar current was also found to be a factor in the experimental device which could be important at low $V_{\mathrm{gs}}$ bias. The other reasons possibly include the use of abrupt doping profiles, and abrupt geometry in the simulation. The limitations of the model include the use of one trap at the mid-gap level, and the lack of electrostatic degradation.

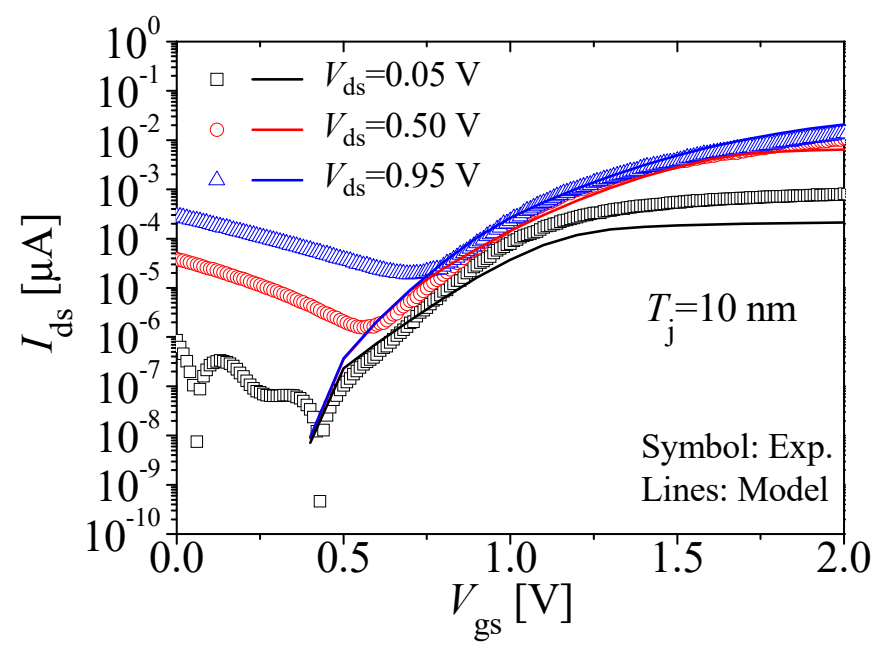

Figure 8. $I_{\mathrm{ds}}$ from the experimental device (symbols) [12] compared with $I_{\mathrm{ds} \_ \text {TAT }}$ calculated from (23). 


\section{Conclusions}

The literature lacks a compact tunable trap-assisted-tunneling model. This work presented a compact TAT model that captured important physical parameters that influence the TAT process including phonon energy, the Huang-Rhys factor, and tunneling masses. The model is divided into three parts. The first part includes the potential model, the second part includes the standard Schenk model equations, and the third part includes the compact implementation of the Schenk model where the TAT tunneling rate is expressed as a Gaussian distribution. The approximated Gaussian distribution is then integrated along the $x$-axis to generate a TAT-generated drain current. The model was tested against the TCAD data and was found to be in reasonable agreement. The model was also tested against $I_{\mathrm{ds}}$ from an experimental device. The model was found to follow the same trend as the experimental device. The result, however, was not truly accurate. This could be due to neglecting the electrostatic degradation caused by the traps which could be important at high bias. Another reason for the inaccuracy could be neglecting effects such as contact resistance in the model. In summary, this work provides a useful contribution within the compact trap-assisted-tunneling modeling domain of TFET devices. The compact implementation part of the model can be generalized for use in any TFET device and can easily be incorporated in the simulation program with integrated circuit emphasis (SPICE) framework. For the completed compact model of the TET for SPICE implementation, series resistance [22], SRH [23], ambipolar, quantum confinement [24], and breakdown models [25] should be added to this proposed model.

Author Contributions: Conceptualization, Y.S.Y. and F.N.; Data curation, Y.S.Y. and F.N.; Formal analysis, Y.S.Y. and F.N.; Investigation, F.N.; Methodology, F.N.; Project administration, Y.S.Y.; Supervision, Y.S.Y.; Visualization, F.N.; Writing—original draft, F.N.; Writing—review and editing, Y.S.Y. All authors have read and agreed to the published version of the manuscript.

Funding: This research was funded by the Ministry of Trade, Industry, and Energy (MOTIE), project number 10054888 and Korea Semiconductor Research Consortium (KSRC) support program for the development of future semiconductor devices.

Acknowledgments: This work was supported by IDEC (EDA tool).

Conflicts of Interest: The authors declare no conflict of interest.

\section{References}

1. Boucart, K.; Ionescu, A. Double-gate tunnel FET with high-א gate dielectric. IEEE Trans. Electron. Devices 2007, 54, 1725-1733. [CrossRef]

2. Flori, G.; Iannaccone, G. Ultra-voltage bilayer graphene tunnel FET. IEEE Electron. Device Lett. 2009, 30, 1096-1098.

3. Datta, S.; Liu, H.; Narayan, V. Tunnel FET Technology: A Reliability Perspective. Microelectron. Reliab. 2014, 54, 861-874. [CrossRef]

4. Alian, A.; Mols, Y.; Bordallo, C.C.M.; Verreck, D.; Verhulst, A.; Vandooren, A.; Rooyackers, R.; Agopian, P.G.D.; Martino, J.A.; Thean, A.; et al. InGaAs tunnel FET with sub-nanometer EOT and sub-60 mV/dec sub-threshold swing at room temperature. Appl. Phys. Lett. 2016, 109, 243502. [CrossRef]

5. Sajjad, R.N.; Chern, W.; Hoyt, J.L.; Antoniadis, D.A. Trap assisted tunneling and its effect on subthreshold swing of tunnel FETs. IEEE Trans. Electron. Devices 2016, 63, 4380-4387. [CrossRef]

6. Sajjad, R.N.; Antoniadis, D. A compact model for tunnel FET for all operating regimes including trap assisted tunneling. In Proceedings of the 2016 74th Annual Device Research Conference (DRC), Newark, DE, USA, 19-22 June 2016.

7. Hurkx, G.A.M.; Klassen, D.B.M.; Knuvers, M.P.G. A new recombination model for device simulation including tunneling. IEEE Trans. Electron. Devices 1992, 39, 331-338. [CrossRef]

8. Jiménez-Molinos, F.; Palma, A.; Gámiz, F.; Banqueri, J.; López-Villanueva, J.A. Physical model for trap-assisted inelastic tunneling in metal-oxide-semiconductor structures. J. Appl. Phys. 2001, 90, 3396-3404. [CrossRef] 
9. Schenk, A.; Sant, S.; Moselund, K.; Riel, H. The impact of hetero-junction and oxide-interface traps on the performance of InAs/Si tunnel FETs. In Proceedings of the 2017 17th International Workshop on Junction Technology, Uji, Japan, 1-2 June 2017.

10. Synopsys. User Manual, Version L-2016.03, Synopsys TCAD Sentaurus; Synopsys: San Jose, CA, USA, 2016.

11. Schenk, A. A model for the field and temperature dependence of Shockley-Read-Hall lifetimes in silicon. Solid-State Electron. 1992, 35, 1585-1596. [CrossRef]

12. Kim, S.W.; Kim, J.H.; Liu, T.K.; Choi, W.Y.; Park, B. Demonstration of L-shaped tunnel field-effect transistors. IEEE Trans. Electron. Devices 2016, 63, 1774-1778. [CrossRef]

13. Najam, F.; Yu, Y.S. Physically consistent method for calculating trap-assisted-tunneling current applied to line tunneling field-effect-transistor. IEEE Trans Electron. Devices 2020, 67, 2106-2112. [CrossRef]

14. Vandenberghe, W.G.; Verhulst, A.S.; Groeseneken, G.; Soree, B.; Magnus, W. Analytical model for point and line tunneling in a tunnel field-effect transistor. In Proceedings of the 2008 International Conference on Simulation of Semiconductors Processes and Devices, Hakone, Japan, 9-11 September 2008.

15. Najam, F.; Yu, Y.S. Investigation of corner effect and identification of tunneling regimes in L-shaped tunnel field-effect-transistor. J. Nanosci. Nanotechnol. 2018, 18, 6578-6583. [CrossRef] [PubMed]

16. Najam, F.; Yu, Y.S. Compact model for L-shaped tunnel field-effect transistor including the 2D region. Appl. Sci. 2019, 9, 3716. [CrossRef]

17. Smets, Q.; Verhulst, A.S.; Simeon, E.; Gundlach, D.; Richter, D.; Collaert, N.; Heyns, M.M. Calibration of bulk trap-assisted tunneling and Shockley-Read-Hall currents and impact on InGaAs tunnel-FETs. IEEE Trans. Electron. Devices 2017, 64, 3622-3626. [CrossRef]

18. Mandurrino, M.; Goano, M.; Vallone, M.; Bertazzi, F.; Ghione, G.; Verzellesi, G.; Meneghini, M.; Meneghesso, G.; Zanoni, E. Semiclassical simulation of trap-assisted tunneling in GaN-based light-emitting diodes. J. Comput. Electron. 2015, 14, 444-445. [CrossRef]

19. Vincent, G.; Chantre, A.; Bois, D. Electric field effect on the thermal emission of traps in semiconductor junctions. J. Appl. Phys. 1972, 50, 5484-5487. [CrossRef]

20. Fleming, R.M.; Seager, C.H.; Lang, D.V.; Campbell, J.M. Injection deep level transient spectroscopy: An improved method for measuring capture rates of hot carriers in semiconductors. J. Appl. Phys 2015, 118, 015703. [CrossRef]

21. Mendenhall, W.; Beaver, R.J.; Beaver, B.M. Introduction to Probability and Statistics Metric Edition, 15th ed.; Cengage Learning US: Boston, MA, USA, 2019.

22. Klassen, F.; Biermans, P.; Velghe, R. The series resistance of submicron MOSFETs and its effect on their characteristics. J. Phys. Colloq. 1988, 49, C4-257-C4-260. [CrossRef]

23. Liu, Y.; Yan, J.; Wang, H.; Han, G. Temperature dependent $I_{\mathrm{DS}}-V_{\mathrm{GS}}$ characteristics of an N-channel Si tunneling field-effect transistor with a germanium source on $\mathrm{Si}(110)$ substrate. J. Semicond. 2014, 35, 024001. [CrossRef]

24. Walke, A.M.; Verhulst, A.S.; Vandooren, A.; Verreck, D.; Simoen, E.; Rao, V.R.; Groeseneken, G.; Collaert, N.; Thean, A.V.Y. Part I: Impact of field-induced quantum confinement on the subthreshold swing behavior of line TFETs. IEEE Trans. Electron. Devices 2013, 60, 4057-4064. [CrossRef]

25. Jung, H.; Dimitrijev, S. Analysis of flat-band-voltage dependent breakdown voltage for $10 \mathrm{~nm}$ double gate. MOSFET. J. Inf. Commun. Converg. Eng. 2018, 16, 43-47.

(C) 2020 by the authors. Licensee MDPI, Basel, Switzerland. This article is an open access article distributed under the terms and conditions of the Creative Commons Attribution (CC BY) license (http://creativecommons.org/licenses/by/4.0/). 